\title{
Survey on QoE/QoS Correlation Models for Video Streaming over Vehicular Ad-hoc Networks
}

\author{
Abdelkader Benmir ${ }^{1}$, Ahmed Korichi $^{2}$, Abdelhabib Bourouis ${ }^{1}$ and \\ Mohammed Alreshoodi ${ }^{3}$ \\ ${ }^{1} \operatorname{ReLa}(\mathrm{CS})^{2}$ Laboratory, Oum El Bouaghi University, Oum El Bouaghi, Algeria \\ ${ }^{2}$ Department of Computer Science, Ouargla University, Ouargla, Algeria \\ ${ }^{3}$ Community College, Qassim University, Unizah, Saudi Arabia
}

\begin{abstract}
Vehicular Ad-hoc Networks (VANETs) are a new emerging technology which has attracted enormous interest over the last few years. It enables vehicles to communicate with each other and with roadside infrastructures for many applications. One of the promising applications is multimedia services for traffic safety or infotainment. The video service requires a good quality to satisfy the end-user known as the Quality of Experience (QoE). Several models have been suggested in the literature to measure or predict this metric. In this paper, we present an overview of interesting researches, which propose QoE models for video streaming over VANETs. The limits and deficiencies of these models are identified, which shed light on the challenges and real problems to overcome in the future.
\end{abstract}

ACM CCS (2012) Classification: Networks $\rightarrow$ Network properties $\rightarrow$ Network manageability

Networks $\rightarrow$ Network performance evaluation $\rightarrow$ Network simulations

Keywords: vehicular ad-hoc networks (VANETs), multimedia services, quality of service (QoS), quality of experience (QoE), quality metrics

\section{Introduction}

Vehicular Ad-hoc Networks (VANETs) represent a rapidly emerging and a particularly challenging class of Mobile Ad Hoc Networks (MANETs) where vehicles communicate [1]. It has a significant interest by car manufacturing industries, academia and government agencies. They have put much joint efforts together towards realizing the concept of vehicular communications in a wide scale. These efforts have led to numerous vehicular communications researches with their associated standardization projects in many countries across the world [2].

VANETs are characterized by:

a) trajectory-based movements with location prediction and time-varying topology,

b) varying number of vehicles with independent or correlated speeds,

c) fast time-varying channel (e.g., signal transmissions can be blocked by buildings),

d) frequent network disconnection due to the movement of vehicles at high speed, and

e) battery power and storage is unlimited [3], [4].

They are self-organizing and self-governing communication networks without any central coordinator [5]. Vehicles can communicate with each other (V2V) or with the Road Side Units (RSU) which are a fixed infrastructure along the roadside (V2I) [6] as shown in Figure 1 [7]. To ensure communication, vehicles use wireless communication equipment which is either short-range or medium-range [8], [9]. For instance, VANETs currently use the Dedicated Short Range Communication technology (DSRC) which is the IEEE 802.11p standard [10], [11], [12]. A VANET architecture is presented in Figure 1.

The main objective of VANET is achieving safe and comfortable driving. It can provide a 
very wide variety of applications in the field of transportation. Typically, applications are categorized as road safety, traffic coordination, and infotainment applications [13], [14], [15], [16]. These applications of VANET mostly need to send multimedia data for communication, which still remains a great challenge on Quality of Services (QoS) and Quality of Experiences

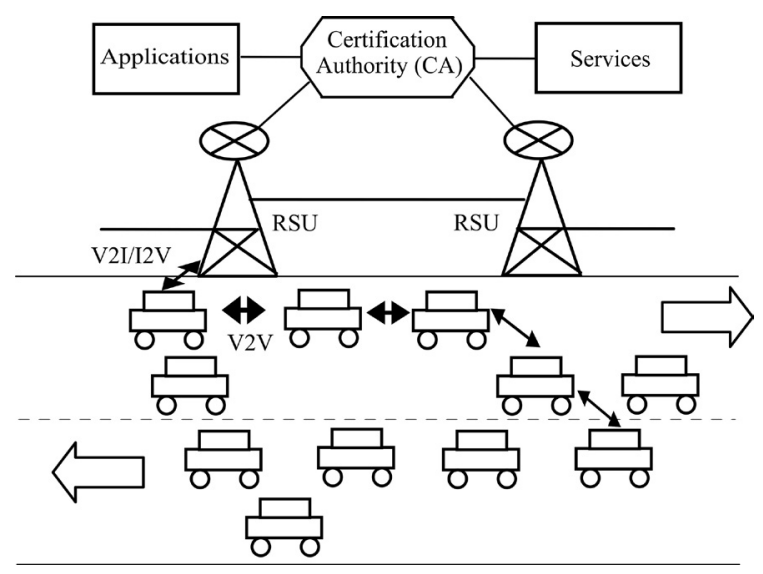

Figure 1. VANET architecture [7].

(QoE) issues due to the nature of VANET.

The QoS is only focused on service requirements that need to be met by the network [17]. It does not include the user and context factors for quality evaluation. The QoE puts emphasis on the degree of satisfaction of users by the offered service [18], [19]. Several researches have been conducted in QoE modeling, monitoring and management. The QoS is objective but the QoE is subjective. Even though QoS and QoE measurements are quite different, they have a high degree of correlation. In the literature, a number of studies have focused on identifying the relationships between QoS and QoE for multimedia services.

This paper presents a comprehensive review and a thorough analysis of recent and pertinent research related to QoS and QoE correlation models for video transmission over VANET. The rest of the paper is organized as follows. In Section 2, video streaming over vehicular networks is highlighted. In Section 3, QoE applications in video streaming are presented. Section 4 introduces the influence factors of QoE in video streaming. Section 5 is a literature re- view of QoE models for video streaming over Wireless Network, Mobile Network, MANET and VANET. In Section 6, limits and deficiencies of QoE models for video streaming over VANET are identified and discussed, which opens wide doors of challenges and issues to deal with in the future. Finally, conclusions and perspectives are given in Section 7.

\section{Video Streaming Over Vehicular Networks}

The video streaming is a client-server paradigm where interactive media data of streaming applications such as video on demand, IP Television (IPTV), video conferencing, e-learning and videogames are exchanged, shared and consumed [20]. Video streaming over vehicular networks is a need for many applications. These applications can generally be grouped into two categories: safety applications and non-safety applications as shown in Figure 2. We summarize the main applications of these categories in the next paragraphs [21], [22], [23], [24].

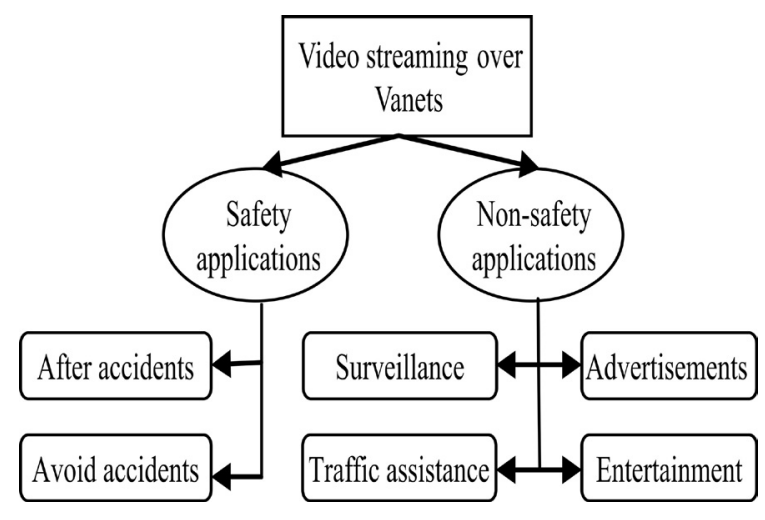

Figure 2. Video streaming applications over vehicular network.

\subsection{Video in Safety Applications}

The aim of safety applications is saving the life of drivers and passengers. The video can play an important role to avoid accidents by showing dangerous situations such as collision risk, vehicle lane change, overtaking vehicle, stopped vehicle, work zone, pedestrian crossing and low bridge. It can also be helpful even 
after accidents when offering collision recording, notifications for other vehicles, telemedicine, medication identification, help in guiding emergency rescuers and authorities, investigation support for accident liability.

\subsection{Video in Non-safety Applications}

The aim of non-safety applications is traffic assistance, surveillance, entertainment and advertisements. In traffic assistance, the video can assist driver decisions and give a clear overview of the road traffic conditions to better trajectory planning by avoiding congestions and closed roads. It can show him dangers on the road and weather information. The video can be also used to prevent anti-social behaviour such as crimes, robbery, irritating people inside/outside vehicles. It can also help authorities to identify vehicles or pedestrians they are searching for. Besides all these, video can be used in comfort and entertainment. Vehicles can exchange video information like multi-player games for entertainment, live video stream of both sides, video conferencing and video changing. Roadside commerce can use video in advertisements of services like nearest parking lots, gas stations, shopping malls, hotels and fast food restaurants.

Video streaming is considered as one of the challenging issues in VANET due to highly dynamic network topology, high mobility, frequent connectivity interruption and requirements of Quality of Experience (QoE) and Quality of Service (QoS), typical for multimedia applications. The most challenging points are achieving high rates of delivery ratio with less delay in available bandwidth and offering a good quality [21], [22], [23], [24].

\section{QoE Applications in Video Streaming}

The theory of quality of experience (QoE) has been widely used to represent user perception. For network service evaluation, a number of models and tools have been proposed in QoE modeling, monitoring (assessment) and management with the exploration of many influencing factors.
In the QoE monitoring, we measure or estimate the QoE of video streaming using dedicated tools with the consideration of the influencing factors. To monitor QoE, two schemes were developed which are subjective test and objective QoE monitoring. In the QoE management, videos are prepared and encoded with proper quality levels in order to maximize the user's QoE. In the QoE modeling, models are developed to measure or predict the QoE as closely as possible to the QoE perceived by the end users. It aims to model the relationship between different QoE influencing factors. These models can be adopted in QoE monitoring [25].

In this paper, we focus on the pertinent proposed QoE models of video streaming in VANETs found in the literature. In the next section, we present the main QoE factors of video streaming and review the corresponding proposed models.

\section{Influence Factors of QoE in Video Streaming}

Quality of Experience (QoE) is a set of human-centric metrics that captures the overall acceptability or unacceptability of the service or application by end users, which includes the end-to-end factors [26]. The QoE is affected by various factors of experience that are grouped in three main categories: human, system, and context influence factors [26], [27]. The influence factors of QoE in video streaming are summarized in Figure 3.

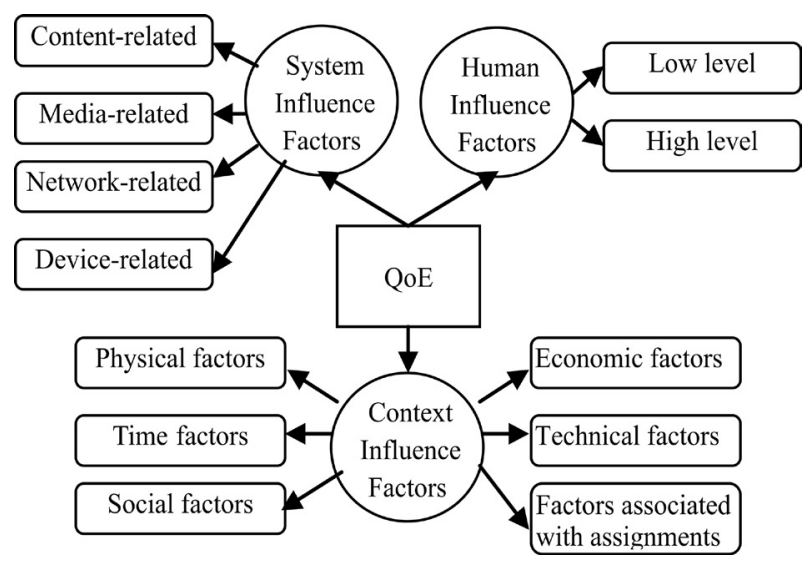

Figure 3. Influence factors of $\mathrm{QoE}$ in video streaming. 


\subsection{Human Influence Factors (H.I.Fs)}

Human Influence Factors are any property or characteristic of a human. They could be divided into two subgroups. The first is the low-level influence factors such as age, gender, personality and mood. The second is the high-level influence factors such as socio-economic conditions, educational background, needs, previous experience and life stage [27].

They are poorly understood and are taken into account in a very limited set of studies [28] [29]. We outline in Table 1, some interesting research projects that studied the influence of human factors on QoE in different domains.

\subsection{System Influence Factors (S.I.Fs)}

System Influence Factors refer to properties and characteristics that determine the technically produced quality of an application or service. They could be classified into content-related, media-related, network-related and device-related factors.

- The content-related S.I.Fs consider the content of the video itself like high-motion or low motion, 2D or 3D.

- The media-related S.I.Fs refer to media configuration factors, such as encoding, resolution, sampling rate, frame rate, and media synchronization.

- Network-related S.I.Fs refer to data transmission over a network. The main network characteristics are bandwidth, delay, jitter, loss and error rates and distributions, and throughput.

- Device-related S.I.Fs refer to the visual interface that displays the video to the user.

A summary of research efforts devoted to the study of the influence of system factors on QoE in diverse domains is given in Table 2.

\subsection{Context Influence Factors (C.I.Fs)}

They consider the environmental factors associated with the user. Their classification includes six sub-groups [27]:

- physical factors,

- time factors,

- social factors,

- economic factors,

- factors associated with assignments, and

- technical factors.

Table 3 presents some research papers which studied the influence of context factors on $\mathrm{QoE}$ in different domains.

Table 1. Human influence factors.

\begin{tabular}{|c||c|c|c|}
\hline Author & Human Influence Factors & Level & Domain \\
\hline \hline Kara [30] & Subject's prior knowledge & High-level & Auto stereoscopic glasses-free 3D video \\
\hline Hyder [31] & Gender (male/female) & Low-level & Virtual acoustic environments \\
\hline Mccoll [32] & Facial expressions and gender & Low-level & Web-video sales \\
\hline Msakni [33] & Male/female, age & Low-level & Lab-based videos \\
\hline Murray [34] & Gender, age & Low-level & Videos sequences \\
\hline Rodriguez [35] & Personal and cultural traits & High-level & Lab-based videos \\
\hline Guntuku [36] & Preference & High-level & Video streaming services \\
\hline Zhu [37] & Gender and age & Low-level & Lab-based videos \\
\hline Chen [38] & User's emotions & High-level & Streaming video \\
\hline Scott [29] & Gender, age & Low-level & Lab-based videos \\
\hline Scott [29] & Personality, culture, nationality & High-level & Lab-based videos \\
\hline Oche [39] & Gender & Low-level & VANET multimedia service \\
\hline
\end{tabular}


Table 2. System influence factors.

\begin{tabular}{|c|c|c|c|c|c|}
\hline \multirow{2}{*}{ Author } & \multicolumn{4}{|c|}{ System Influence Factors } & \multirow{2}{*}{ Domain } \\
\hline & Network-related & Media-related & Content-related & Device-related & \\
\hline Han [40] & $\begin{array}{c}\text { Throughput, } \\
\text { bitrates }\end{array}$ & No & No & User equipment & $\begin{array}{l}\text { Wireless } \\
\text { network }\end{array}$ \\
\hline Xing [41] & & $\begin{array}{l}\text { Start-up latency, } \\
\text { interruption ratio, } \\
\text { average playback } \\
\text { quality }\end{array}$ & No & No & VANET \\
\hline Msakni [33] & Bitrates & No & $\begin{array}{l}\text { Video type (soccer } \\
\text { and documentary), } \\
\text { Video cotenant }\end{array}$ & $\begin{array}{l}\text { Video display } \\
\text { resolution, video } \\
\text { display equipment }\end{array}$ & $\begin{array}{c}\text { Lab-based } \\
\text { videos }\end{array}$ \\
\hline Msakni [42] & No & No & $\begin{array}{l}\text { Video type, video } \\
\text { length }\end{array}$ & No & $\begin{array}{c}\text { Lab-based } \\
\text { videos }\end{array}$ \\
\hline Pokhrel [43] & $\begin{array}{l}\text { Packet loss rate, } \\
\text { packet loss, } \\
\text { burstiness, jitter }\end{array}$ & No & No & No & IPTV \\
\hline De Felice [44] & \begin{tabular}{|c|} 
Node densities, \\
end-to-end distances, \\
Packet Delivery \\
Ratio (PDR)
\end{tabular} & No & No & No & $\begin{array}{c}\text { Live } \\
\text { video } \\
\text { streams in } \\
\text { VANET }\end{array}$ \\
\hline Quan [45] & & $\begin{array}{c}\text { Start-up delay, } \\
\text { Playback freezing }\end{array}$ & No & No & VANET \\
\hline Song [46] & Bitrates & Encoding parameters & $\begin{array}{l}\text { Video content } \\
\text { characteristics }\end{array}$ & $\begin{array}{l}\text { Device display } \\
\text { resolution }\end{array}$ & $\begin{array}{l}\text { Mobile } \\
\text { video } \\
\text { service }\end{array}$ \\
\hline Lina Zhu [47] & $\begin{array}{c}\text { Nodes speed, nodes } \\
\text { number }\end{array}$ & No & No & No & \begin{tabular}{|c|} 
VANET \\
video \\
streaming
\end{tabular} \\
\hline Mocanu [48] & No & $\begin{array}{c}\text { Bit rate, percentage } \\
\text { of I-frames lost, } \\
\text { percentage of I,P,B } \\
\text { frames lost, } \\
\text { sad (sum of absolute } \\
\text { differences), number } \\
\text { of bursts }\end{array}$ & No & No & \begin{tabular}{|l} 
Digital \\
terrestrial \\
television
\end{tabular} \\
\hline Zhu [37] & No & Compression bitrates & Video genre & No & $\begin{array}{c}\text { Lab-based } \\
\text { videos }\end{array}$ \\
\hline Chen [38] & No & $\begin{array}{c}\text { Buffer ratio, } \\
\text { average bitrates }\end{array}$ & No & No & $\begin{array}{c}\text { Streaming } \\
\text { video }\end{array}$ \\
\hline
\end{tabular}

Table 3. Context influence factors.

\begin{tabular}{|c||c|c|}
\hline Author & Context Influence Factors & Domain \\
\hline \hline Pyykko [49] & Physical factors and time factors & Mobile (3D) television \\
\hline Han [40] & Environment factors & Wireless Network \\
\hline Msakni [42] & Day time & Lab-based videos \\
\hline Zhu [37] & Social context, technical factors & Lab-based videos \\
\hline Seufert [50] & Technical factors & Web video streaming \\
\hline
\end{tabular}


Other factors emerge and are related to the nature of the VANET itself such as large-scale sizes, frequent link disconnections, rapid topology changes and the impact of density and driving environments. These factors are connectivity probability, reliability, availability, link duration, hop count, end to end delay and stability [51], [52], [53].

\section{QoE Models for Video Streaming over VANET}

In QoE studies, several models have been proposed in both wired and wireless networks, some of them were presented and evaluated in [54], [55], [56] and [57]. These models are not well adapted to VANETs since they do not take into account their specificities.

In this section, we present QoE models for video streaming over VANETs. In the literature, to the authors' best knowledge, only few studies propose QoE models for video streaming in VANET. This pushes us to extend the studied models designed for Wireless Networks, Mobile Networks and MANETs to include specificities of VANETs, which is itself a sub class of MANETs [58] and can be formed in pure cellular or mobile network [59].

We begin by presenting some QoE models for video streaming over Wireless Networks, Mobile Networks and MANETs, and then discussing QoE models for video streaming in VANET. In this paper we evaluate only QoE models for video streaming in VANETs. Models designed for other networks will be only presented to enlarge the reader's view.

\subsection{QoE Models for Video Streaming over Wireless Network, Mobile Network and MANETs}

We present some QoE models for video streaming over Wireless Networks, Mobile Networks and MANETs, which could be extended to VANETs by considering their characteristics such as high speed of vehicles on predefined roads that makes the topology highly dynamic. The highly dynamic topology generates frequent network disconnection [58].

\subsubsection{Cerqueira - Wireless Mesh Networks}

Cerqueira et al. in [60], propose an on-the-fly parametric QoE video quality estimator for real-time video streaming applications over Wireless Networks. The model was built to assess the QoE of the video perceived by end-users. It takes as input values a set of parameters related to the video characteristics of an encoder, which correspondingly quantify the video quality. The model is based on statistical learning using Multiple Artificial Neural Networks (MANNs). It is designed to evaluate videos coded in standard MPEG.

To estimate the QoE, the model takes into account the current network conditions and different video parameters (loss rate in I, P and $\mathrm{B}$ frames, total loss rate, GoP length, and motion and complexity levels).

Four successive stages were required to implement the Multi QoE approach in a networking system, including Wireless Mesh Networks (WMNs):

- Quality-affecting factors,

- Distorted video database generation,

- Subjective Quality Assessment, and

- Learning the quality of the behaviour with MANNs.

\subsubsection{Fung - MANET}

Fung et al. propose a QoE model based on QoS parameters for web-TV streaming service over Peer-to-peer (P2P) in MANETs [61]. Their MANET system uses the Landmark-based routing model, which provides a free-scale and highly dynamic routing functionality. They define three levels of user experience for qualitative measurement:

- QoS Packet Loss level,

- QoS Packet Delivery level, and

- Resource QoS Contribution level.

They use these parameters in exponential and logarithmic models.

A simulation study using OMNeT++ network simulator was conducted. Specifically, the OverSim framework for overlay and peer-to- 
peer networks with a patched INET framework model are adopted in their simulation scenarios. Moreover, at the application level, a P2P live streaming system is used. The physical distribution of mobile nodes is generated randomly. They performed tests in different scenarios.

\subsubsection{Jiang - Wireless Networks}

Jiang et al. propose a QoE prediction model, which incorporates the sender bitrates, dropping probability and frame rate into the QoE calculation [62]. Some parameters in the prediction model are obtained by a nonlinear regression analysis of the QoS parameters. The model is used in their QoE-driven channel allocation scheme for secondary users and cognitive radio networks (CRN) base station (BS).

To evaluate the system performance, they derived an analytical Markov model combining the ON/OFF model of PCs and the service queuing model. They studied the performance of multimedia transmission of images and H.264 videos. They used MATLAB to calculate the QoE and QoS parameters.

\subsubsection{Zhang - Wireless Networks}

Zhang et al. in [63] propose a logarithmic QoE model built upon experiments relating to the content cache management for HTTP ABR streaming. The model is formulated as a constrained convex optimization problem over wireless networks. They adopt a two-step process to solve the snapshot problem. First, using the Lagrange multiplier method to obtain the numerical solution of the set of playback rates for a fixed number of cache copies and characterize the optimal solution analytically. Second, they develop three alternative search algorithms (i.e., exhaustive search, Dichotomous-based search, and variable step-size search) to find the optimal number of cached files. In their paper, they present a simplified QoE model, in which the user experience depends on two system parameters, including the required playback rate and the actual playback rate. The model is used in a QoE-optimal scheme for the individual content cache management in HTTP ABR streaming.

\subsubsection{Sanchez - MANET}

Sanchez et al. in [64] proposed a model to measure the QoE of video streaming over MANETs. The QoE model has been calculated in terms of MOS (Mean Opinion Score), from a parametric model. It allowed them to obtain accurate video-MOS estimations by means of the Packet Loss Rate (PLR) and the video-coding bit-rate $(\mathrm{Br})$ parameters.

For model validation, they used the simulation framework OMNET++ v4.4, with the InetManet framework v2.2. They carried out simulations with different scenarios.

\subsubsection{Bampis - Mobile Network}

Bampis et al. proposed the MultiQE model for QoE prediction in their continuous Quality of Experience prediction engine over mobile networks [65]. Prediction is based on a non-linear autoregressive model with exogenous outputs (NARX) recurrent neural networks. The QoE prediction model is driven by three QoE-aware inputs:

- an objective measure of perceptual video quality,

- rebuffering aware information and

- a memory of prior events affecting QoE (recency).

They trained and tested their method on QoE dataset containing continuous time subjective scores of viewed video content on a mobile device. They used MATLAB to implement the model.

After model training and validation, MultiQoE is used for QoE prediction in real-time without any intervention of real end users. MultiQoE gives scores in terms of MOS. To test Multi QoE, simulation experiments were carried out using Network Simulator 2.34, Evalvid tool, MSU Video Quality Measurement Tool (VQMT) and the Neural Networks was built using MATLAB.

\subsubsection{Wang - LTE Network (Mobile)}

Wang et al. proposed the HTTP Adaptive Streaming (HAS) QoE prediction methods based on multi-segment and multi-rate features 
of HAS with data mining over LTE networks [66]. They designed two sets of methodologies to evaluate the HAS QoE, including regression and classification. In the regression method, they proposed the evolved Peak Signal-toNoise Ratio (ePSNR) model using differential PSNR (dPSNR) statistics as the segment features to estimate HAS QoE. In the classification method, they proposed the improved weighted $k$-nearest neighbours ( $\mathrm{WkNN}$ ) by using dynamic weighted mapping according to the position of video chunk to meet the dynamic segment and rate features of HAS.

In order to train and test these methods, they built a real-time HAS video-on-demand (VOD) system in LTE network and did subjective test in different video scenes encoded in H.264 format. With the MOS, the regression and classification methods were trained to predict the HAS QoE.

In Table 4, we summarize the evaluated aspects of studied models and in Table 5 their technical aspects.
To apply these models in VANETs, several specific features must be considered such as the high mobility of vehicles, topology fast changes, frequent network disconnections and V2V communications. For mobile networks, QoE models must take into consideration the fast handover and geolocation of vehicles.

To apply these models in VANETs, several specific features must be considered such as the high mobility of vehicles, topology fast changes, frequent network disconnections and V2V communications. For mobile networks, QoE models must take into consideration the fast handover and geolocation of vehicles.

\subsection{QoE Models for Video Streaming over VANET}

In this section, we present the QoE models for video streaming over VANETs. We start by describing these models and then proceed to their evaluation.

Table 4. Evaluated aspects of studied QoE models for video streaming over Wireless Network, Mobile Network and MANETs.

\begin{tabular}{|c|c|c|c|c|c|c|c|c|}
\hline \multirow{2}{*}{ Author } & \multicolumn{4}{|c|}{ System Influence Factors } & \multirow{2}{*}{ H.I.Fs } & \multirow{2}{*}{ C.I.Fs } & \multirow{2}{*}{$\begin{array}{c}\text { QoE } \\
\text { prediction }\end{array}$} & \multirow{2}{*}{$\begin{array}{l}\text { Mapping } \\
\text { function }\end{array}$} \\
\hline & Network & Media & Content & Device & & & & \\
\hline Cerqueira [60] & No & $\begin{array}{l}\text { Frames } \\
\text { losses }\end{array}$ & $\mid \begin{array}{c}\text { Motion and } \\
\text { complexity } \\
\text { levels }\end{array}$ & No & No & No & $\begin{array}{c}\text { Multiple } \\
\text { Artificial } \\
\text { Neural } \\
\text { Network }\end{array}$ & MOS \\
\hline Fung [61] & \begin{tabular}{||c|} 
Packet Loss, \\
Packet Delivery, \\
Video Packet \\
Size,
\end{tabular} & & Resolution & l & / & $\begin{array}{c}\text { Resource } \\
\text { Contribution }\end{array}$ & $\begin{array}{c}\text { Mathematical } \\
\text { model }\end{array}$ & MOS \\
\hline Jiang [62] & No & $\begin{array}{c}\text { Sender bitrates, } \\
\text { dropping } \\
\text { probability, } \\
\text { frame rate }\end{array}$ & No & No & No & No & Analytical & MOS \\
\hline Zhang [63] & No & \begin{tabular}{|c|} 
Playback \\
rate, actual \\
playback rate
\end{tabular} & No & No & No & No & Logarithmic & MOS \\
\hline \begin{tabular}{|c|} 
Sanchez-Iborra \\
{$[64]$}
\end{tabular} & $\begin{array}{l}\text { Packet Loss } \\
\text { Rate }\end{array}$ & $\begin{array}{c}\text { video-coding } \\
\text { bit-rate }\end{array}$ & l & l & / & & Mathematical & MOS \\
\hline Bampis [65] & l & $\begin{array}{l}\text { Video quality, } \\
\text { rebuffering }\end{array}$ & / & l & l & $\begin{array}{l}\text { Prior events } \\
\text { affecting } \\
\text { QoE }\end{array}$ & $\begin{array}{l}\text { NARX } \\
\text { (ANN) }\end{array}$ & / \\
\hline Wang [66] & No & $\begin{array}{l}\text { Bit rates, } \\
\text { video } \\
\text { segment }\end{array}$ & No & No & No & No & $\begin{array}{l}\text { Statistical } \\
\text { model }\end{array}$ & MOS \\
\hline
\end{tabular}


Table 5. Technical aspects of studied QoE models for video streaming over Wireless Network, Mobile Network and MANETs.

\begin{tabular}{|c|c|c|c|c|c|c|c|c|}
\hline Author & $\begin{array}{l}\text { Communi- } \\
\text { cation V2V } \\
\end{array}$ & $\begin{array}{c}\text { Infra- } \\
\text { structure }\end{array}$ & \begin{tabular}{|c|c|}
$\begin{array}{c}\text { Video } \\
\text { reference }\end{array}$ \\
\end{tabular} & Validation tools & $\begin{array}{c}\text { Model } \\
\text { mobility }\end{array}$ & $\begin{array}{c}\text { Environ- } \\
\text { ment } \\
\end{array}$ & Protocol & $\begin{array}{c}\text { Video } \\
\text { service } \\
\end{array}$ \\
\hline $\begin{array}{c}\text { Cerqueira } \\
{[60]}\end{array}$ & 1 & Yes & No & \begin{tabular}{|c|} 
MATLAB \\
+ Network \\
Simulator 2.34
\end{tabular} & I & 1 & 1 & $\begin{array}{c}\text { Video } \\
\text { streaming }\end{array}$ \\
\hline Fung [61] & No & & No & $\begin{array}{c}\text { Omnet++, } \\
\text { OverSim, INET }\end{array}$ & 1 & No & \begin{tabular}{|c|} 
Propose a \\
protocol
\end{tabular} & $\begin{array}{c}\text { P2P live video } \\
\text { streaming }\end{array}$ \\
\hline Jiang [62] & l & / & l & $\begin{array}{c}\text { Markov } \\
\text { Models } \\
+ \text { MATLAB }\end{array}$ & / & / & l & $\begin{array}{c}\text { HTTP } \\
\text { adaptive bit } \\
\text { rate streaming }\end{array}$ \\
\hline Zhang [63] & l & Yes & l & l & l & / & l & $\begin{array}{c}\text { HTTP } \\
\text { adaptive bit } \\
\text { rate streaming }\end{array}$ \\
\hline $\begin{array}{c}\text { Sanchez- } \\
\text { Iborra [64] }\end{array}$ & $\begin{array}{l}\text { Device } \\
\text { to device }\end{array}$ & Yes & No & $\begin{array}{l}\text { Omnet++ v4.4 + } \\
\text { InetManet V2.2 }\end{array}$ & I & l & $\begin{array}{l}\text { BATMAN, } \\
\text { OLSR, } \\
\text { AODV }\end{array}$ & $\begin{array}{c}\text { Video- } \\
\text { streaming }\end{array}$ \\
\hline $\begin{array}{c}\text { Bampis } \\
{[65]}\end{array}$ & 1 & Yes & l & MATLAB & & l & l & $\begin{array}{c}\text { Video on } \\
\text { mobile device }\end{array}$ \\
\hline Wang [66] & 1 & 1 & No & $\begin{array}{c}\text { Lab simulation+ } \\
\text { statistical } \\
\text { validation }\end{array}$ & 1 & / & / & $\begin{array}{l}\text { Video-on- } \\
\text { demand }\end{array}$ \\
\hline
\end{tabular}

\subsubsection{Asefi}

Asefi et al. proposed a quality of service (QoS) model based on video distortion in their cross-layer path selection scheme for video streaming over VANETs in an urban environment [67]. In the proposed scheme, the video is streamed from a RSU to a destination vehicle via multi-hop communication. The objective in the path selection is to minimize application layer video distortion. The distortion is the summation of encoding distortion, distortion due to delay and distortion due to packet loss in VANET. To calculate the distortion, in their model they used Distortion of packet due to encoding, Rate of encoder video packet, Rate of video packet and End-to-end delay factors.

To evaluate their model, a mathematical model for an urban architecture was used. They streamed a 20 seconds of MPEG encoded video with 30 frames per second rate in dense and sparse scenarios. They used the PSNR to measure the performance of the routing protocol.

The first problem in this model is the assumption of a wide availability of roadside units. The issues of ad hoc communication are mostly neglected. The model can be applied only for streaming of all the videos from one RSU to the destination vehicle. They didn't consider channels and external interference. In the validation, they fixed the average arrival rate of the vehicles.

\subsubsection{Pham}

Pham et al. proposed a QoE model for video streaming for VANETs [68]. The QoE is evaluated as MOS at the destination vehicle. They used the Pseudo-Subjective Quality Assessment (PSQA) tool to obtain the MOS. They studied the impact of loss rate (LR) and mean loss burst size (MLBS) on MOS. The model is combined with OLSR protocol for the path selection from the source to the destination.

They evaluated the performance of the proposed mechanism using Network Simulator (NS) version 2.35. The Bonn Motion tool was used to create the Manhattan Grid scenario. They compared the performance of their protocol with AODV and MPOLSR protocols for video streams. The number of video streams varied from 1 to 8 . The video is requested by the vehicle from one of four servers. 
The proposed model has some limits. They used only two parameters: loss rate (LR) and Mean loss burst size (MLBS). They did not consider the MOS of every intermediate node but only that of two-hop nodes. PSQA tool takes only network-oriented parameters. Moreover, they did not take into consideration the path lifetime.

\subsubsection{Wolfinger}

Starting from the fact that in IPTV, the main influencing factor of QoE for users is TV channels availability, Wolfinger et al. introduced a new model to measure the QoE in [69]. It is an analytical model that predicts the blocking probability of TV channels for channel-switching-induced and handover-induced blocking events in IPTV services in VANETs.

They proposed two models, one for all IPTV users and the other for individual users. The first model is used to predict the QoE when a channel is demanded (watched) by more than one user. The second model is used to predict the QoE when a channel is demanded (watched) by individual users.

In their paper, they focused on the model related to individual users. In their proposed model, they calculate channel-switching and handover in a cell of the access network.

To validate their model, they implemented the model and applied a numerical case study scenario to obtain the QoE. They compared the analytical results to simulation results and found that they are close enough.

The proposed model has some limits:

- They did not take in account V2V communications.

- They assumed that a high number of installed RSU cover the entire road.

- They did not measure the quality of the video received by the end-user.

- They assumed that vehicles are driving at a constant speed in their lane.

- They assumed that the maximum bandwidth available in a cell and the data rate required to send to each TV channel are constant.

\subsubsection{Yaacoub}

Yaacoub et al. proposed a QoE model measured in terms of the PSNR and of two other parameters that depend on the video characteristics as QoE metrics [70]. The model used in their techniques is based on grouping the vehicles into cooperative clusters. In each cluster, the cluster head receives the video over long-range cellular links and multicasts it to vehicles in its cluster over IEEE 802.11p.

To validate their proposition, simulations in MATLAB were implemented in a highway environment. They used four scalable video coded (SVC) sequences. Three scenarios were investigated in these simulations:

- the video is transmitted to a moving vehicle via RSUs,

- the video is transmitted to a moving vehicle using LTE BS, and

- booth RSUs and LTE BS transmit the video.

The proposed QoE model has some limits:

- Video distortions caused by compression method at the source are neglected.

- PSNR needs the original video to be calculated and is not well correlated to the subjective quality that end users perceive.

- They did not explain how to calculate the QoE parameters.

- They did not specify which video characteristics represent the QoE parameters.

- Their proposed scheme needs LTE BS and RSU to be deployed on the side of highway.

- The scheme is applicable only with infrastructure, which is the initial source of video stream.

- The solution is not bandwidth-efficient because of the large amount of data sent and lost due to packet losses.

\subsubsection{Quadros}

In [71], authors propose a model to calculate QoE as a function of Mean Square Error and distortion. They used this model in their protocol called quality driven beaconless multi-cri- 
teria video streaming management protocol for VANETs.

The QoE model is designed for MPEG standard which defines that the Group of Pictures (GoP) is composed of a combination of three frame types, namely I (Intra), P (Predictive), and B (Bidirectionally predictive) frames. QoE in this model is affected by frame types (I, P, B), codec configurations, and losses. It is used to select the best next hop and to switch to other routes as soon as a lower quality is identified. They consider $40 \mathrm{~dB}$ as the highest video quality, and the lowest video quality has values below $20 \mathrm{~dB}$.

To validate their proposition, they conduct a simulation using SUMO, EvalVid (A Video Quality Evaluation Tool-set) and Network Simulator version 2.33. To demonstrate the impact of their model in delivering QoE aware video flows in VANETs, they used a geographic routing approach called VIRTUS, Dynamic Backbone Assisted (DBA) MAC protocol and Beaconless Routing protocol (BLR) are used for comparison.

The proposed model has the following limits:

- It did not take into account the routing path lifetime and multiple flows.

- It could not be used with RSU.

- In the model, the video must be streamed only from vehicles.

- It did not consider context and human factors.

- It is applicable only in highway environment.

- It is used only for MPEG standard.

\subsubsection{Immich}

Immich et al. proposed a self-adaptive forward error correction based proactive error recovery mechanism and QoE-driven mechanism to shield video transmissions over VANETs (SHIELD) [72]. SHIELD uses several video characteristics and specific VANET details to safeguard real-time video streams against packet losses. It combines network density, signal-to-noise ratio, packet loss rate, and the vehicle's position to better protect the video sequences and enhance the QoE. The mechanism uses Unequal Error Protection (UEP) because not all video packets have the same importance to ensure the final video quality. To improve these issues, SHIELD adopts a Hierarchical Fuzzy System (HFS) that estimates the QoE by combining network quality indicator and video characteristics. To establish network quality indicator, it evaluates four parameters which are SNR, PLR, the network density and the position of vehicles. To establish video characteristics indicator, it evaluates six parameters, which are temporal intensity, spatial complexity, frame size, frame type, resolution and macro block detail.

To validate the proposed mechanism, they conducted simulations for urban and highway environments. The Network Simulator 3 (NS-3) was used to conduct the experiments. All videos were encoded with H.264, GoP length of 19:2, where three different resolutions were used, namely 1080p, 720p, and SVGA. For each resolution, 10 videos were chosen to be transmitted. To send videos, they used Evalvid Tool. The mobility traces were generated using the Simulation of Urban MObility (SUMO). For the routing protocol, Cross-Layer, Weighted, Position-based Routing (CLWPR) were used.

The model has some limits:

- If the infrastructure is available, it could be used, but the optimizations are to be performed only on the communication between the vehicles.

- They used few videos and network parameters.

- Device related and context influence factors are not considered.

- The impact of delay in QoE, which has a great effect, is not studied.

\subsubsection{Sarwar}

Sarwar et al. modeled the QoE as a mapped function to MOS [73]. The proposition consists of an adaptive selection of application QoS parameters and an IVT handover decision mechanism for providing seamless imagery effect and enhance user's multimedia perception. The suggested architecture can provide a sustainable channel rate based selection of content source, media resolution, and codec to improve the perceived visual quality. They proposed a model 
of Handover/Handoff management to avoid a necessary handover. The parameters used in the QoE model are the media codec, frame rate, display screen size and the user requested frame rate.

The model has been tested by simulations under Inet and Veins frameworks of OMNET ++ and SUMO traffic simulator, in urban environment. They used these parameters: compression algorithms such as MPEG-2, MPEG-4, H.264, and SVC, packet drop ratio (PDR), average packet delay (APD), content source, media codec, and resolution.

The main limit of this model is the use of one hop vehicle-RSU communication and there is no multi-hop V2V communication which is the main characteristic of VANETs. The model could be applied only in urban environment. No information about the QoE model parameters is given.

\subsubsection{Oche}

Oche et al. proposed an objective real-time multimedia service QoE prediction model based on a multivariate statistical approach, in conjunction with regression analysis that estimates perceived multimedia service quality as a function of aggregated QoE influencing weighted parameters [39]. To structure the proposed QoE prediction model, they segment the multimedia/VANET distribution network into a framework of three quality optimization components, taking into account the service source quality, the network resource constraint and the human factor in determining the overall QoE. The QoE is estimated as a weighted sum of the QoE influencing parameters. The influencing parameters in their work are divided into two categories:

- technique parameters which are for application layer: frame rate, and bit rate, for network layer: packet loss, throughput and delay,

- no-technique parameters (context), which are user's physical environmental (city/ highway/rural), user terminal resolution and user gender.

To validate the model, they used a statistical method in highway and city environments with infrastructure RSU to support $\mathrm{V} 2 \mathrm{~V}$ and
V2I communications. They also used Markov chains Monte Carlo (MCMC) simulation to generate gender, environmental and user terminal resolution. As technique parameters, they used their previous results of simulation under NS2 [74].

This model suffers from the problem that parameters in the ordered logit model are obtained through the maximum likelihood technique (point estimate) where the estimations are only valid for current network connectivity matrix. This means that they did not consider the topology change, which is the main feature of VANETs. They did not use any mobility. The model did not consider the encoding distortion at the source and packet loss in the area of the wired segment of the network connection path. For validation, they made statistical methods instead of tests using video streaming. Furthermore, the values of jitter, buffer, video content coder, routing protocol, gender, environment and user terminal resolution, are not taken from simulation, but are randomly generated.

\subsubsection{Fei Sun}

Fei Sun et al. proposed a QoE evaluation model established by using empirical data for vehicular video streaming performed in cellular networks [75]. They also developed a mathematical relationship between the streaming bit rate and caching storage space of base station of cellular networks. Then, they formulated a distributed caching management for vehicular video streaming as a constrained optimization problem and solved it using the generalized reduced gradient method. In their system, the video is divided into different blocks, and then some adjustments are made to the bitrates of each video segment, with considerations on the limited caching space.

The video is streamed via cellular networks from sever to vehicles. The mobility of users's vehicles in cellular networks is modeled as Hyper Erlang distribution. Their QoE evaluation model is based on Weber Fechner law, which follows logarithmic laws. The QoE influence factors used are bit rate and videos types.

They used MATLAB to implement their proposed QoE centric distributed caching approach in cellular networks. They evaluate their work in different scenarios by changing the number 
of cells, caching space size and vehicle's density.

The model suffers from the following limits:

- The model is applicable only in VANETs video streaming under cellular networks.

- They suppose that the user subscribes to one video.

- There is no V2V communication.

- They studied few influence factors.

- The user's location and mobility are pre-calculated and estimated when users subscribe to video services.

- It cannot be used in scenarios when vehicles send videos.

- There is a need to consider geolocation data for the vehicular multimedia content distribution in cellular networks.

\section{Discussion and Future Issues}

The Table 6 and Table 7 summarize the aspects evaluated by models discussed above, as well as how many different aspects were evaluated in each model and technical aspect of studied models. Different angles were taken when it comes to factors used in the models, method of prediction and mapping, some technical aspects.

The state of the art of QoE modeling of video streaming over VANETs is reviewed, evaluated and criticized. The proposed models have many limits and drawbacks, which open a wide door of challenges and issues.

\subsection{Model's Limits}

The study of existing models has shown several limits, which can be summarized as follows.

\subsubsection{Restrained Number of Models}

There are only few proposed models dealing with QoE over VANETs. This interesting research area did not get attention it deserves. Consequently, existing models are far from being perfect. Thorough studies and much effort are to be devoted in order to propose more appropriate models.

\subsubsection{Models Formulation}

The study of existing and recent models reveals that the relationship between QoE and its influencing factors is complex and nonlinear. This relationship has not been fully understood to allow developing of an accurate and representative model for QoE. The use of machine learning techniques could give an added value to this domain. It provides a theoretical and methodological framework to quantify the relationship between influencing factors to predict the QoE.

\subsubsection{Influence Factors}

Studied models show that only a limited number of influencing parameters are considered. This cannot provide an accurate prediction of the QoE for end-users. These models neglect the effect of important parameters like human and context influence factors, except for the previously discussed Oche model. Moreover, Oche model considers only three parameters: gender, terminal resolution and user's physical environment. The influence factors have not been fully investigated or quantitatively modeled. This is due to their mathematical scaling, which is still a very challenging task. The focus of formulated models is put on network-related and media-related parameters neglecting other influencing factors as content-related, device-related, and human and context influence factors.

\subsubsection{Unrealistic VANET Scenarios}

The QoE video streaming models were simulated in unrealistic scenarios. Abstract mobility models, simplified signal propagation models, no consideration of obstacles, which block the radio signal and hence will cause high video packet loss. Models need to be re-evaluated using realistic vehicular scenarios.

\subsubsection{Performance Evaluation}

The proposed models seem to have good performance parameters according to suggested scenarios in their databases. Unfortunately, there is no performance evaluation and comparison of different models in other databases. To evaluate them objectively, they must be tested under different databases with various conditions. 
Table 6. Evaluated aspects of studied QoE models of video streaming over VANETs.

\begin{tabular}{|c|c|c|c|c|c|c|c|c|c|}
\hline \multirow{2}{*}{ Author } & \multicolumn{4}{|c|}{ System Influence Factors } & \multirow{2}{*}{ H.I.Fs } & \multirow{2}{*}{ C.I.Fs } & \multirow{2}{*}{\begin{tabular}{|c|} 
QoE \\
prediction
\end{tabular}} & \multirow{2}{*}{$\begin{array}{l}\text { Mapping } \\
\text { function }\end{array}$} & \multirow{2}{*}{$\begin{array}{l}\text { Video } \\
\text { codec }\end{array}$} \\
\hline & Network & Media & Content & Device & & & & & \\
\hline Asefi [67] & $\begin{array}{c}\text { packet rate, } \\
\text { delay }\end{array}$ & $\begin{array}{c}\text { encoding } \\
\text { packet loss, } \\
\text { encoder } \\
\text { packet rate }\end{array}$ & No & No & No & No & No & No & I \\
\hline Pham [68] & $\begin{array}{l}\text { Loss rate, } \\
\text { mean loss } \\
\text { burst size }\end{array}$ & No & No & No & No & No & PSQA & MOS & l \\
\hline $\begin{array}{c}\text { Wolfinger } \\
\text { [69] }\end{array}$ & $\begin{array}{l}\text { Channels } \\
\text { witching, } \\
\text { hand over }\end{array}$ & No & No & No & No & No & Analytical & \begin{tabular}{|c|} 
one \\
blocking per \\
hour to be \\
acceptable \\
+ switching \\
greater than \\
0.2 will be \\
unacceptable \\
for the user
\end{tabular} & / \\
\hline $\begin{array}{c}\text { Yaacoub } \\
{[70]}\end{array}$ & No & $\begin{array}{l}\text { Frame rate, } \\
\text { spatial } \\
\text { resolution, } \\
\text { spatial, } \\
\text { temporal } \\
\text { variances }\end{array}$ & No & No & No & No & Analytical & $\begin{array}{c}Q=0 \text { best } \\
\text { quality } \\
Q=100 \\
\text { worst quality }\end{array}$ & \begin{tabular}{|c} 
Two \\
layers \\
scalable \\
video \\
coded \\
(SVC)
\end{tabular} \\
\hline $\begin{array}{l}\text { Quadros } \\
\text { [71] }\end{array}$ & No & $\begin{array}{l}\text { Frame types, } \\
\text { codec } \\
\text { configurations }\end{array}$ & No & No & No & No & Analytical & $\begin{array}{c}40 \mathrm{~dB}= \\
\text { highest } \\
\text { video quality } \\
20 \mathrm{~dB}< \\
\text { lowest video } \\
\text { quality }\end{array}$ & MPEG-4 \\
\hline $\begin{array}{l}\text { Immich } \\
\text { [72] }\end{array}$ & $\mid$\begin{tabular}{|c} 
SNR, PLR, \\
network \\
density, \\
position of \\
vehicles
\end{tabular} & $\begin{array}{c}\text { Frame size, } \\
\text { frame type, } \\
\text { resolution, } \\
\text { macroblock } \\
\text { detail }\end{array}$ & \begin{tabular}{|c|} 
motion \\
activity: \\
temporal \\
intensity, \\
spatial \\
complexity
\end{tabular} & No & No & No & $\begin{array}{l}\text { Fuzzy } \\
\text { System }\end{array}$ & I & $\begin{array}{l}\text { Codec } \\
\text { H.264 }\end{array}$ \\
\hline $\begin{array}{c}\text { Sarwar } \\
\text { [73] }\end{array}$ & No & $\begin{array}{c}\text { Media } \\
\text { codec, frame } \\
\text { rate }\end{array}$ & No & $\begin{array}{l}\text { Screen } \\
\text { size }\end{array}$ & No & No & Analytical & MOS & $\begin{array}{c}\text { MPEG-2, } \\
\text { MPEG-4, } \\
\text { H.264, } \\
\text { SVC }\end{array}$ \\
\hline Oche [39] & $\mid \begin{array}{c}\text { Packet loss, } \\
\text { throughput } \\
\text { delay }\end{array}$ & $\begin{array}{c}\text { Frame rate, } \\
\text { bit rate }\end{array}$ & No & $\begin{array}{c}\text { Terminal } \\
\text { resolution }\end{array}$ & gender & \begin{tabular}{|c|} 
user's \\
physical \\
environment \\
(city, and \\
highway/ \\
rural) \\
\end{tabular} & Statistical & MOS & MPEG-4 \\
\hline Sun [75] & No & Bit rate & $\begin{array}{l}\text { Videos } \\
\text { types }\end{array}$ & No & No & No & $\begin{array}{c}\text { Weber } \\
\text { Fechner law } \\
\text { (logarithmic } \\
\text { laws) }\end{array}$ & MOS & / \\
\hline
\end{tabular}


Table 7. Technical aspect of studied QoE models of video streaming over VANETs.

\begin{tabular}{|c|c|c|c|c|c|c|c|c|}
\hline Author & $\begin{array}{l}\text { Communi- } \\
\text { cation V2V }\end{array}$ & \begin{tabular}{c|} 
Infra- \\
structure
\end{tabular} & $\begin{array}{c}\text { Video } \\
\text { reference }\end{array}$ & Validation tools & $\begin{array}{c}\text { Model } \\
\text { mobility }\end{array}$ & \begin{tabular}{|c|} 
Environ- \\
ment
\end{tabular} & Protocol & $\begin{array}{c}\text { Video } \\
\text { service }\end{array}$ \\
\hline Asefi [67] & No & RSU \& AR & $\begin{array}{c}\text { FR } \\
\text { (PSNR) }\end{array}$ & Analytical & No & $\begin{array}{l}\text { Streets in } \\
\text { an urban }\end{array}$ & $\begin{array}{c}\text { An } \\
\text { enhanceed } \\
\text { greedy } \\
\text { georouting }\end{array}$ & $\begin{array}{c}\text { Video } \\
\text { streaming }\end{array}$ \\
\hline Pham [68] & $\mathrm{V} 2 \mathrm{~V}$ & No & No & $\begin{array}{l}\text { Simulation } \\
\text { (NS2) }\end{array}$ & No & l & $\begin{array}{l}\text { Modified } \\
\text { OLSR }\end{array}$ & $\begin{array}{c}\text { Video } \\
\text { streaming }\end{array}$ \\
\hline \begin{tabular}{|c|} 
Wolfinger \\
[69]
\end{tabular} & No & RSU & No & $\begin{array}{l}\text { Simulation/ } \\
\text { Analytical }\end{array}$ & $\begin{array}{c}\text { Constant } \\
\text { speed }\end{array}$ & Motorways & No & IPTV \\
\hline $\begin{array}{c}\text { Yaacoub } \\
{[70]}\end{array}$ & $\mathrm{V} 2 \mathrm{~V}$ & $\begin{array}{c}\text { RSUs and } \\
\text { LTE BS }\end{array}$ & $\begin{array}{c}\text { FR } \\
\text { (PSNR) }\end{array}$ & $\begin{array}{l}\text { Simulation } \\
\text { (MATLAB) }\end{array}$ & Random & Highway & No & $\begin{array}{c}\text { Video } \\
\text { streaming }\end{array}$ \\
\hline $\begin{array}{c}\text { Quadros } \\
\text { [71] }\end{array}$ & $\mathrm{V} 2 \mathrm{~V}$ & No & No & $\begin{array}{c}\text { Simulation } \\
\text { (NS2) }\end{array}$ & Realistic & Highway & $\begin{array}{c}\text { No (new pro- } \\
\text { tocol) }\end{array}$ & $\begin{array}{c}\text { Video } \\
\text { streaming }\end{array}$ \\
\hline $\begin{array}{l}\text { Immich } \\
\text { [72] }\end{array}$ & $\mathrm{V} 2 \mathrm{~V}$ & No & No & $\begin{array}{l}\text { Simulation } \\
\text { (NS3) }\end{array}$ & I & $\begin{array}{c}\text { Urban and } \\
\text { highway }\end{array}$ & \begin{tabular}{|c|} 
Cross-Layer, \\
Weighted, \\
Position- \\
based \\
Routing \\
(CLWPR) \\
\end{tabular} & $\begin{array}{c}\text { Video } \\
\text { streaming }\end{array}$ \\
\hline $\begin{array}{c}\text { Sarwar } \\
\text { [73] }\end{array}$ & $\mathrm{V} 2 \mathrm{~V}$ & RSU & No & $\begin{array}{c}\text { Simulation } \\
(\mathrm{OMNET}++)\end{array}$ & l & Urban & $\begin{array}{c}\text { WAVE short } \\
\text { message } \\
\text { protocol } \\
\text { (WSMP) }\end{array}$ & $\begin{array}{l}\text { In-vehicle } \\
\text { telescreen } \\
\text { (IVT) }\end{array}$ \\
\hline \begin{tabular}{|c|} 
Oche [39], \\
{$[74]$}
\end{tabular} & $\mathrm{V} 2 \mathrm{~V}$ & RSU & No & $\begin{array}{l}\text { Simulation } \\
\text { (NS2) }\end{array}$ & / & $\begin{array}{l}\text { Highway } \\
\text { and city }\end{array}$ & AODV & IPTV \\
\hline Sun [75] & No & $\begin{array}{c}\text { Base } \\
\text { station } \\
\text { (cellular } \\
\text { network) }\end{array}$ & No & $\begin{array}{l}\text { Simulation } \\
\text { (MATLAB) }\end{array}$ & $\begin{array}{c}\text { Hyper- } \\
\text { Erlang } \\
\text { distribution }\end{array}$ & No & No & $\begin{array}{l}\text { VANET video } \\
\text { streaming } \\
\text { under cellular } \\
\text { networks }\end{array}$ \\
\hline
\end{tabular}

\subsubsection{Radio Access Technologies}

VANET can use different radio access technologies (WiFi, WiMAX, LTE, 4G and 5G). Only one studied model is based on cellular networks but does not take into consideration the V2V communication. All the reviewed models neglect other technologies as WiFi, cellular networks or LTE.

\subsubsection{Routing Protocols}

Various underlying routing protocols were used in these models. Nevertheless, the choice of a particular protocol has never been justified. In our opinion, the routing protocol affects the QoE and must be considered as a parameter.

\subsection{Future Issues}

The following issues constitute some of the promising research directions towards developing new models to measure the QoE of video streaming over VANETs.

\subsubsection{Influence Factors}

The fundamental challenge of the quality predictor model is the choice of appropriate influencing factors to predict the QoE for all videos in different environments and scenarios. There is still an open issue on how to select the leading component from all possible system, context and human Influence factors in the design of QoE models. 


\subsubsection{Influence Factors' Relations}

The influence factors have not been fully investigated or quantitatively modeled. This is because their mathematical scaling is still a very challenging task. Therefore, it is an open field of research to propose mathematical models or machine learning based models.

\subsubsection{Influence Factors' Nature}

The established models focus on measurable factors such as throughput, delay, jitter, or loss rate, and neglect immeasurable factors such as gender, environment and period of viewing, which have a great influence on QoE. To evaluate appropriately the QoE, models must consider all major influencing factors.

\subsubsection{Realistic VANET Scenarios}

The video streaming quality models must be simulated in realistic scenarios. Especially, the vehicular communications with wireless shadowing and fading channel deterioration, in addition to diverse environments: urban, rural and highway with obstacles and real mobility models.

\subsubsection{Database for Performance Evaluation}

The proposed models are tested in their own databases. It is desirable to elaborate test databases containing videos with different features and characteristics to validate the proposed models. It is also desirable to build a subjective database covering potential QoE influencing factors, video types, VANET characteristics and user characteristics. This database will serve as a reference to test the proposed QoE models.

\subsubsection{Radio Access Technologies}

The QoE video models over VANETs should be able to measure the QoE of various VANET architectures as with or without RSU, with or without cellular mobile networks and so forth. VANET can use different radio access technologies (WiFi, WiMAX, LTE, 4G and 5G) and credible models must take into consideration all technologies used.

\subsubsection{Video Type and Codec}

Different video types and codecs are developed like auto stereoscopic 3DTV (AS3DTV), 4K ultra high definition (4K-UHD), and super HDTV (SHDTV). Models should be easily customized to deal with various video coding.

\subsubsection{Models Standardization}

The study of QoS and QoE of video service in VANETs appeared many years ago, but till now there has been no standardization of influencing factors, methods of measurement or prediction of QoE. Most research focus on formulating models and trying to find a relation between the QoE and factors. It is important to address research to standardize the QoE over VANET for video service.

\subsubsection{Routing Protocol for Video Streaming}

The underlying routing protocols for VANETs should be evaluated and compared to find the most suitable one for video service over VANETs. In addition, new proposed routing protocols for video streaming over VANETs are of great interest.

\section{Conclusion}

Quantifying the QoE-QoS relationship is an extremely challenging task and the ultimate judge of telecommunication service quality. In this paper, various issues regarding the QoE of video streaming applications over VANETs are presented. We identified and summarized the most interesting factors that influence the end user QoE of video streaming. The influencing factors were classified in various categories and the recent research in this area has been thoroughly reviewed. Some QoE models of video streaming over wireless, ad-hoc, mobile and MANETs have been discussed. A special focus is put on VANETs where all the pertinent QoE models of video streaming found in the literature were presented, analysed and evaluated. Finally, the paper identified some challenges that still require innovative solutions for modeling the video quality in order to improve the 
QoE perceived by users of a video streaming service. With regard to the challenges of QoE/ QoS correlation modeling, there is still a need to identify and better understand many QoE influencing factors for a given type of service and to find out how they influence each other.

\section{References}

[1] J. Harri et al., "Mobility Models for Vehicular Ad hoc Networks: a Aurvey and Taxonomy", IEEE Communications Surveys \& Tutorials, vol. 11, no. 4, pp. 19-41, 2009.

https://doi.org/10.1109/SURV.2009.090403

[2] H.-J. Fischer, "Standardization and Harmonization Activities Towards a Global C-ITS", in Vehicular ad hoc Networks, Springer, 2015, pp. 23-36.

https://doi.org/10.1007/978-3-319-15497-8_2

[3] F. J. Martinez et al., "A Survey and Comparative Study of Simulators for Vehicular Ad hoc Networks (VANETs)", Wireless Communications and Mobile Computing, vol. 11, no. 11, pp. 813-828, 2011.

https://doi.org/10.1002/wcm.859

[4] F. Li and Y. Wang, "Routing in Vehicular Ad hoc Networks: a Survey", IEEE Vehicular technology magazine, vol. 2, no. 2, pp. 12-22, 2007. https://doi.org/10.1109/MVT.2007.912927

[5] S. Zeadally et al., "Vehicular Ad hoc Networks (VANETS): Status, Results, and Challenges", Telecommunication Systems, vol. 50, no. 4, pp. 217-241, 2012.

https://doi.org/10.1007/s11235-010-9400-5

[6] E. Ahmed and H. Gharavi, "Cooperative Vehicular Networking: a Survey", IEEE Transactions on Intelligent Transportation Systems, vol. 19, no. 3, pp. 996-1014, 2018.

https://doi.org/10.1109/TITS.2018.2795381

[7] S. K. Bhoi and P. M. Khilar, "Vehicular Communication: a Survey", IET Networks, vol. 3, no. 3, pp. 204-217, 2013.

http://dx.doi.org/10.1049/iet-net.2013.0065

[8] S. Boussoufa-Lahlah et al., "Geographic Routing Protocols for Vehicular Ad hoc NETworks (VANETs): a Survey", Vehicular Communications, vol. 11, pp. 20-31, 2018. https://doi.org/10.1016/j.vehcom.2018.01.006

[9] J. Wang et al., "Networking and Communications in Autonomous Driving: a Survey", IEEE Communications Surveys \& Tutorials, 2018. https://doi.org/10.1109/COMST.2018.2888904

[10] Z. Lu et al., "A Survey on Recent Advances in Vehicular Network Security Trust and Privacy", IEEE Transactions on Intelligent Transportation
Systems, pp. 1-17, 2018

https://doi.org/10.1109/TITS.2018.2818888

[11] G. Karagiannis et al., "Vehicular Networking: A Survey and Tutorial on Requirements, Architectures, Challenges, Standards and Solutions", IEEE communications surveys \& tutorials, vol. 13 , no. 4 , pp. 584-616, 2011.

https://doi.org/10.1109/SURV.2011.061411.00019

[12] F. D. Da Cunha et al., "Data Communication in VANETs: Protocols, Applications and Challenges", Ad Hoc Networks, vol. 44, pp. 90-103, 2016. https://doi.org/10.1016/j.adhoc.2016.02.017

[13] H. Hartenstein and L. P. Laberteaux, "A Tutorial Survey on Vehicular Ad hoc Networks", IEEE Communications magazine, vol. 46, no. 6, pp. 164-171, 2008.

https://doi.org/10.1109/MCOM.2008.4539481

[14] S. Al-Sultan et al., "A Comprehensive Survey on Vehicular Ad hoc Network", Journal of network and computer applications, vol. 37, pp. 380-392, 2014.

https://doi.org/10.1016/j.jnca.2013.02.036

[15] T. L. Willke et al., "A Survey of Inter-vehicle Communication Protocols and Their Applications", IEEE Communications Surveys \& Tutorials, vol. 11, no. 2, pp. 3-20, 2009. https://doi.org/10.1109/SURV.2009.090202

[16] F. A. Silva et al., "Vehicular Networks: A New Challenge for Content-delivery-based Applications", ACM Computing Surveys (CSUR), vol. 49, no. 1, pp. 11, 2016. https://doi.org/10.1145/2903745

[17] I. T. U.-T. R. P.10/G.100-Amendment, "Vocabulary for performance and quality of service", International Telecommunication Union, November 2017, pp. 1-50.

[18] R. Schatz et al., "From Packets to People: Quality of Experience as a New Measurement Challenge", in Data traffic monitoring and analysis, Springer, 2013, pp. 219-263.

https://doi.org/10.1007/978-3-642-36784-7_10

[19] M. Fiedler et al., "A Generic Quantitative Relationship Between Quality of Experience and Quality of Service", IEEE Network, vol. 24, no. 2, pp. 36-41, 2010.

https://doi.org/10.1109/MNET.2010.5430142

[20] T. Phakathi et al., "Quality of Service of Video Streaming in Vehicular Adhoc Networks: Performance Analysis", in Proc. of IEEE Int. Conference on Computational Science and Computational Intelligence (CSCI), 2016, pp. 505-509. https://doi.org/10.1109/CSCI.2016.0172

[21] A. Vinel et al., "Video Transmission Over IEEE 802.11 p: Real-world Measurements", in Proc. of IEEE Int. Conference on Communications Workshops (ICC), 2013.

https://doi.org/10.1109/ICCW.2013.6649286 
[22] K. Z. Ghafoor, "Video Streaming over Vehicular Ad Hoc Networks: A Comparative Study and $\mathrm{Fu}-$ ture Perspectives", ARO - The Scientific Journal of Koya University, vol. 4, no. 2, pp. 25-36, 2016. http://dx.doi.org/10.14500/ARO.10128

[23] E. C. Eze et al., "Advances in Vehicular Ad-hoc Networks (VANETs): Challenges and Road-map for Future Development", International Journal of Automation and Computing, vol. 13, no. 1, pp. 1-18, 2016.

https://doi.org/10.1007/s11633-015-0913-y

[24] A. Rasheed et al., "Vehicular Ad Hoc Network (VANET): a Survey, Challenges, and Applications", in Vehicular Ad-Hoc Networks for Smart Cities, Springer, pp. 39-51, 2017.

https://doi.org/10.1007/978-981-10-3503-6_4

[25] L. Skorin-Kapov et al. "A Survey of Emerging Concepts and Challenges for QoE Management of Multimedia Services", ACM Transactions on Multimedia Computing, Communications, and Applications (TOMM), vol. 14, no. 2s, pp. 29:129:13, 2018.

https://doi.org/10.1145/3176648

[26] K. Brunnström et al., "Qualinet White Paper on Definitions of Quality of Experience", Lausanne, 2013.

[27] M. Yang et al., "Survey on QoE Assessment Approach for Network Service", IEEE Access, vol. 6, pp. 48374-48390, 2018.

https://doi.org/10.1109/ACCESS.2018.2867253

[28] J. B. Husić et al., "What Factors Influence the Quality of Experience for WebRTC Video Calls?", in Proc. of IEEE 40th Int. Convention on Information and Communication Technology, Electronics and Microelectronics (MIPRO), 2017.

https://doi.org/10.23919/MIPRO.2017.7973463

[29] M. J. Scott et al., "Do Personality and Culture Influence Perceived Video Quality and Enjoyment?", IEEE Transactions on Multimedia, vol. 18, no. 9, pp. 1796-1807, 2016.

https://doi.org/10.1109/TMM.2016.2574623

[30] P. A. Kara et al., "Distortions in QoE Measurements of Ubiquitous Mobile Video Services Caused by the Preconceptions of Test Subjects", in Proc. of IEEE/IPSJ 12th Int. Symposium on Applications and the Internet, 2012.

https://doi.org/10.1109/SAINT.2012.74

[31] M. Hyder et al., "Are QoE requirements for Multimedia Services Different for Men and Women? Analysis of Gender Differences in Forming QoE in Virtual Acoustic Environments", in B. S. Chowdhry, F. K. Shaik, D. M. A. Hussain and M. A. Uqaili (Eds.), Emerging Trends and Applications in Information Communication Technologies, vol. 281, Berlin, Heidelberg: Springer, 2012. https://doi.org/10.1007/978-3-642-28962-0_20
[32] R. McColl and Y. Truong, "The Effects of Facial Attractiveness and Gender on Customer Evaluations During a Web-video Sales Encounter", Journal of Personal Selling \& Sales Management, vol. 33, no. 1, pp. 117-128, 2013.

https://doi.org/10.2753/PSS0885-3134330110

[33] H. G. Msakni and H. Youssef, "Is QoE Estimation Based on QoS Parameters Sufficient for Video Quality Assessment?", in Proc. of IEEE 9th Int. Conference on Wireless Communications and Mobile Computing Conference (IWCMC), 2013. https://doi.org/10.1109/IWCMC.2013.6583615

[34] N. Murray et al., "Age and Gender Influence on Perceived Olfactory \& Visual Media Synchronization", in Proc. of IEEE 9th Int. Conference on Multimedia and Expo (ICME), 2013. https://doi.org/10.1109/ICME.2013.6607467

[35] D. Z. Rodríguez et al., "Video Quality Assessment in Video Streaming Services Considering user Preference for Video Content", IEEE Transactions on Consumer Electronics, vol. 60, no. 3, pp. 436-444, 2014.

https://doi.org/10.1109/TCE.2014.6937328

[36] S. C. Guntuku et al., "Modelling the Influence of Personality and Culture on Affect and Enjoyment in Multimedia", in Proc. of IEEE Int. Conference on Affective Computing and Intelligent Interaction (ACII), 2015.

https://doi.org/10.1109/ACII.2015.7344577

[37] Y. Zhu et al., "Understanding the Role of Social Context and user Factors in Video Quality of Experience", Computers in Human Behavior, vol. 49, pp. 412-426, 2015.

https://doi.org/10.1016/j.chb.2015.02.054

[38] M. Chen et al., "User Intent-oriented Video QoE with Emotion Detection Networking", in Proc. of IEEE Int. Conference on Global Communications Conference (GLOBECOM), 2016. https://doi.org/10.1109/GLOCOM.2016.7842364

[39] M. Oche et al., "Multivariate Statistical Approach for Estimating QoE of Real-time Multimedia Applications in Vehicular ITS Network", Computer Communications, vol. 104, pp. 88-107, 2017. https://doi.org/10.1016/j.comcom.2016.12.022

[40] B. Han et al., "QoE Model Based Optimization for Streaming Media Service Considering Equipment and Environment Factors", Wireless Personal Communications, vol. 66, no. 3, pp. 595-612, 2012.

https://doi.org/10.1007/s11277-012-0739-7

[41] M. Xing and L. Cai, "Adaptive Video Streaming with Inter-vehicle Relay for Highway VANET Scenario", in Proc. of IEEE Int. Conference on Communications (ICC), 2012.

https://doi.org/10.1109/ICC.2012.6364143

[42] H. G. Msakni and H. Youssef, "Impact of NonQoS Related Parameters on Video QoE", in Proc. of 4th Int. Workshop on Perceptual Quality of 
Systems, 2013.

http://dx.doi.org/10.21437/PQS.2013-10

[43] J. Pokhrel et al., "Estimation of QoE of Video Traffic Using a Fuzzy Expert System", in Proc. of IEEE Int. Conference on Consumer Communications and Networking Conference (CCNC), 2013. https://doi.org/10.1109/CCNC.2013.6488450

[44] M. De Felice et al., "A Distributed Backbone-based Framework for Live Video Sharing in VANETs", in Proc. of the 11th ACM symposium on Performance evaluation of wireless ad hoc, sensor, \& ubiquitous networks, 2014. https://doi.org/10.1145/2653481.2653486

[45] W. Quan et al., "Social Cooperation for Information-centric Multimedia Streaming in Highway VANETs", in Proc. of IEEE 15th Int. Symposium on a World of Wireless, Mobile and Multimedia Networks (WoWMoM), 2014.

https://doi.org/10.1109/WoWMoM.2014.6918992

[46] W. Song and D. W. Tjondronegoro, "Acceptability-based QoE Models for Mobile Video", IEEE Transactions on Multimedia, vol. 16, no. 3, pp. 738-750, 2014. https://doi.org/10.1109/TMM.2014.2298217

[47] L. Zhu et al., "On Dynamic Video Source Decision in VANETs: an On-demand Clustering Approach", International Journal of Distributed Sensor Networks, vol. 11, no. 6, pp. 436810, 2015.

https://doi.org/10.1155/2015/436810

[48] D. C. Mocanu et al., "No-reference Video Quality Measurement: Added Value of Machine Learning", Journal of Electronic Imaging, vol. 24, no. 6, pp. 061208, 2015.

https://doi.org/10.1117/1.JEI.24.6.061208

[49] S. Jumisko-Pyykkö and T. Utriainen, "A Hybrid Method for Quality Evaluation in the Context of Use for Mobile (3D) Television", Multimedia Tools and Applications, vol. 55, no. 2, pp. 185-225, 2011.

https://doi.org/10.1007/s11042-010-0573-4

[50] M. Seufert et al., "Unperturbed Video Streaming QoE under Web Page Related Context Factors", in Proc. of IEEE 9th Int. Conference on Quality of Multimedia Experience (QoMEX), 2017. https://doi.org/10.1109/QoMEX.2017.7965675

[51] G. Li et al., "Adaptive Quality-of-service-based Routing for Vehicular Ad hoc Networks with Ant Colony Optimization", IEEE Transactions on Vehicular Technology, vol. 66, no. 4, pp. 3249-3264, 2017.

https://doi.org/10.1109/TVT.2016.2586382

[52] M. H. Eiza et al., "Situation-aware QoS Routing Algorithm for Vehicular Ad hoc Networks", IEEE Transactions on Vehicular Technology, vol. 64, no. 12, pp. 5520-5535, 2015. https://doi.org/10.1109/TVT.2015.2485305
[53] Y. Sun et al., "An Adaptive Routing Protocol Based on QoS and Vehicular Density in Urban VANETs", International Journal of Distributed Sensor Networks, vol. 11, no. 6, pp. 631092, 2015.

https://doi.org/10.1155/2015/631092

[54] M. Alreshoodi and J. Woods, "Survey on QoE/ QoS Correlation Models for Multimedia Services", International Journal of Distributed and Parallel Systems, vol. 4, no. 3, pp. 53-72, 2013. https://doi.org/10.5121/ijdps.2013.4305

[55] S. Aroussi and A. Mellouk, "Survey on Machine Learning-based QoE-QoS Correlation Models", in Proc. of IEEE Int. Conference on Computing, Management and Telecommunications (ComManTel), 2014.

https://doi.org/10.1109/ComManTel.2014.6825604

[56] P. Juluri et al., "Measurement of Quality of Experience of Video-on-demand Services: A Survey", IEEE Communications Surveys \& Tutorials, vol. 18, no. 1, pp. 401-418, 2016.

https://doi.org/10.1109/COMST.2015.2401424

[57] T. Zhao et al., "QoE in Video Transmission: A User Experience-driven Strategy", IEEE Communications Surveys \& Tutorials, vol. 19, no. 1, pp. 285-302, 2017.

https://doi.org/10.1109/COMST.2016.2619982

[58] P. Ranjan and K. K. Ahirwar, "Comparative Study of Vanet and Manet Routing Protocols", in Proc. of the Int. Conference on Advanced Computing and Communication Technologies (ACCT 2011), 2011.

[59] C. Xu et al., "QoE-driven User-centric VoD Services in Urban Multihomed P2P-based Vehicular Networks", IEEE Transactions on Vehicular Technology, vol. 62, no. 5, pp. 2273-2289, 2013. https://doi.org/10.1109/TVT.2012.2228682

[60] E. Cerqueira et al., "A Parametric QoE Video Quality Estimator for Wireless Networks", in Proc. of IEEE Int. Globecom Workshops (GC Wkshps), 2012.

https://doi.org/10.1109/GLOCOMW.2012.6477766

[61] K.-C. Fung and Y.-K. Kwok, "A QoE Based Performance Study of Mobile Peer-to-peer Live Video Streaming", in Proc. of IEEE 13th Int. Conference on Parallel and Distributed Computing, Applications and Technologies (PDCAT), 2012. https://doi.org/10.1109/PDCAT.2012.139

[62] T. Jiang et al., "QoE-driven Channel Allocation Schemes for Multimedia Transmission of Priority-based Secondary Users Over Cognitive Radio Networks", IEEE Journal on Selected Areas in Communications, vol. 30, no. 7, pp. 1215-1224, 2012. https://doi.org/10.1109/JSAC.2012.120807

[63] W. Zhang et al., "QoE-driven Cache Management for HTTP Adaptive Bit Rate Streaming Over 
Wireless Networks", IEEE Transactions on Multimedia, vol. 15, no. 6, pp. 1431-1445, 2013. https://doi.org/10.1109/TMM.2013.2247583

[64] R. Sanchez-Iborra and M.-D. Cano, "An Approach to a Cross Layer-based QoE Improvement for MANET Routing Protocols", Network Protocols and Algorithms, vol. 6, no. 3, pp. 18-34, 2014.

https://doi.org/10.5296/npa.v6i3.5827

[65] C. G. Bampis et al., "Continuous Prediction of Streaming Video QoE Using Dynamic Networks", IEEE Signal Processing Letters, vol. 24, no. 7, pp. 1083-1087, 2017. https://doi.org/10.1109/LSP.2017.2705423

[66] F. Wang et al., "HAS QoE Prediction Based on Dynamic Video Features with Data Mining in LTE Network", Science China Information Sciences, vol. 60, no. 4, pp. 042404:1-042404:14, 2017.

https://doi.org/10.1007/s11432-015-1044-3

[67] M. Asefi et al., "A Cross-layer Path Selection Scheme for Video Streaming Over Vehicular Adhoc Networks", in Proc. of IEEE Int. Conference on Vehicular Technology Conference Fall (VTC 2010-Fall), 2010. https://doi.org/10.1109/VETECF.2010.5594547

[68] T. A. Q. Pham et al., "QoE-aware Routing for Video Streaming Over VANETs", in Proc. of IEEE 80th Vehicular Technology Conference (VTC Fall), 2014. https://doi.org/10.1109/VTCFall.2014.6966141

[69] B. E. Wolfinger et al., "A Validated Analytical Model for Availability Prediction of IPTV Services in VANETs", Electronics, vol. 3, no. 4, pp. 689-711, 2014.

http://dx.doi.org/10.3390/electronics3040689

[70] E. Yaacoub et al., "QoE Enhancement of SVC Video Streaming Over Vehicular Networks Using Cooperative LTE/802.11 P Communications", IEEE Journal of Selected Topics in Signal Processing, vol. 9, no. 1, pp. 37-49, 2015. https://doi.org/10.1109/JSTSP.2014.2330343

[71] C. Quadros et al., "Beacon-less Video Streaming Management for VANETs Based on QoE and Link-quality", in Proc. of IFIP/IEEE Int. Symposium on Integrated Network Management (IM), 2015.

https://doi.org/10.1109/INM.2015.7140292

[72] R. Immich et al., "Shielding Video Streaming Against Packet Losses Over VANETs", Wireless Networks, vol. 22, no. 8, pp. 2563-2577, 2016. https://doi.org/10.1007/s11276-015-1112-z

[73] G. Sarwar et al., "Quality of Service and Mobility Aware In-vehicle Telescreen Service Architecture", Technical gazette, vol. 24, no. 1, pp. 177-185, 2017.

https://doi.org/10.17559/TV-20150819110655
[74] M. Oche et al., "Network Centric QoS Performance Evaluation of IPTV Transmission Quality Over VANETs", Computer Communications, vol. 61, pp. 34-47, 2015. https://doi.org/10.1016/j.comcom.2014.12.001

[75] F. Sun et al., "A QoE Centric Distributed Caching Approach for Vehicular Video Streaming in Cellular Networks", Wireless Communications and Mobile Computing, vol. 16, no. 12, pp. 1612-1624, 2016. https://doi.org/10.1002/wcm.2636

Received: July 2018 Revised: January 2019 Accepted: February 2019 
Contact addresses:

Abdelkader Benmir $\operatorname{ReLa}(\mathrm{CS})^{2}$ Laboratory

Oum El Bouaghi University

04000 Oum El Bouaghi

Algeria

e-mail: benmir.aek@univ-ouargla.dz

Ahmed Korichi

Department of Computer Science

Ouargla University

30000 Ouargla

Algeria

e-mail: ahmed.korichi@univ-ouargla.dz

Abdelhabib Bourouis

$\operatorname{ReLa}(\mathrm{CS})^{2}$ Laboratory

Oum El Bouaghi University

04000 Oum El Bouaghi

Algeria

e-mail: a.bourouis@univ-oeb.dz

Mohammed Alreshoodi Community College

Qassim University

52571 Unizah

Saudi Arabia

e-mail: mo.alreshoodi@qu.edu.sa
Abdelkader Benmir is a PhD student in Computer Science at the University of Oum El Bouaghi (Algeria). He received both his "Ingénieur" and "Magister" degree in Computer Science from the University of Biskra in 2002 and 2006, respectively. He is "Maitre Assistant A" at the University of Ouargla (Algeria) since 2009. His doctoral research project focuses on QoS and QoE in Vehicular Ad Hoc Networks.

AHMED Korichi received his engineer degree in Computer Science from the University of Batna (Algeria) in 1987 and his Master and PhD degrees in Computer Science from the University of Batna, Algeria in 2001 and 2009, respectively. He obtained his "HDR" degree from the University of Biskra, Algeria in 2010. He joined the Department of Computer Science and Information Technology at the University of Ouargla, Algeria. Since 2001, Dr. Ahmed Korichi has been conducting research in the areas of network design and performance, modelling and simulation, QoS, wireless ad hoc networks, and vehicular networks. He has reviewed contributions in several scientific journals.

ABDElHaBiB Bourouis received his "Ingénieur" degree in Computer Science from the University of Constantine (Algeria) in 1999, his "Magister" and "Doctorat en Sciences" degrees in Computer Science in 2003 and 2011, respectively, both from the University of Batna (Algeria). He obtained his "HDR" degree from the University of Biskra in 2015. He has been "Maitre de Conférences A" at the University of Oum El Bouaghi (Algeria) since 2003. His research interests include artificial intelligence, model driven engineering, cryptology, performance evaluation, and parallel and distributed simulation.

Mohammed Alreshood received a B.Sc. degree in Computer Science in 2005 from the Qassim University, Buraydah, Saudi Arabia and a M.Sc. (Hons.) in Computer Networking and Information in 2011 from the University of Essex, UK. He subsequently successfully completed his $\mathrm{PhD}$ at the University of Essex in 2016. He is currently a lecturer at Qassim University. His research interests are: computer networking in general, along with quality of experience and quality of service for multimedia streaming. 